\section{Naturschutzaspekte beim Anbau von Biomasse}

\author{
Der erwartete Anbau von Biomasse bereitet einigen Naturschützern Sorge. \\ Die Schäden durch eine zu intensive Landwirtschaft sollen sich nicht wieder- \\ holen. Die Integration von Naturschutzzielen in die Produktion von Biomasse \\ ist aber möglich.
}

W Von Johann Köppel, Wolfgang Peters und Christian Schultze ährend der Bioenergie eine blühende ökonomische Zukunft vorausgesagt wird, betrachten Naturschützer den erwarteten Boom der Anbaubiomasse bislang mit gemischten Gefühlen. Eine im Sinne der Nachhaltigkeit konzipierte Strategie der Energiegewinnung aus Biomasse muss daher auch die Ziele des Naturschutzes so weit wie möglich integrieren und mögliche Zielkonflikte minimieren. Zunehmend rücken auch die positiven Synergien zwischen Biomassenutzung und Naturschutz ins Blickfeld.

Die Biomasse kann in Zukunft mit circa 15 Prozent erhebliche Anteile der Energieversorgung in Deutschland decken - dabei sind bereits die Restriktionen berücksichtigt, die sich aus begrenzten Flächen und strengen Umweltanforderungen ergeben (1). Denn die Bioenergie sollte die Bemühungen um eine umweltverträgliche Landwirtschaft nicht unterlaufen. Zudem fördert die frühzeitige Integration von Naturschutzzielen eine breite Akzeptanz der Bioenergie.

Grundsätzlich besteht auf zwei Feldern Konfliktpotenzial zwischen Naturschutz und Anbaubiomasse. Zum einen besteht eine Flächenkonkurrenz, denn dort, wo Biomasse angebaut wird, kann nicht gleichzeitig vorrangig den Zielen des Naturschutzes nachgekommen werden. Zum anderen bestehen potenzielle Konflikte bezüglich der Art beziehungsweise Intensität der Nutzung der Anbauflächen. Naturschutz erfordert eine angepasste Form der Nutzung, die auch auf den landwirtschaftlich genutzten Flächen Naturschutzfunktionen gewährleistet.

\section{- Flächenpotenzial und Flächenkonkurrenz}

Die landwirtschaftliche Flächennutzung wird in naher Zukunft tief greifende Veränderungen erfahren. Die Agrarreformbeschlüsse der EU, die
Minderung der Exportsubventionierung als Folge der WTO-Beschlüsse, die stetige Effizienzsteigerung in der Landwirtschaft sowie sinkende Einwohnerzahlen führen dazu, dass die erforderlichen Produktionsmengen und damit die Flächenansprüche der Landwirtschaft für Nahrungsund Futtermittel sinken. Diese frei werdenden Flächen könnten genauso für den Energiepflanzenanbau wie für Vorrangflächen im Naturschutz genutzt werden.

Für das Flächenpotenzial des Biomasseanbaus bedeutet das, dass in einem gewissen Maße Naturschutzflächen zu berïcksichtigen sind, will man den Zielen einer umwelt- und naturverträglichen Produktion gerecht werden. So sollte auf keinen Fall wertvolles Grünland für den Anbau von Energiepflanzen genutzt werden und bestehende Ackerflächen zum Teil zu Gehölzstreifen und Sukzessionsflächen entwickelt werden.

Das Projekt „Stoffstromanalyse zur nachhaltigen energetischen Nutzung von Biomasse" hat anhand von Szenariorechnungen die entsprechenden Restriktionen für die Anbauflächen für Biomasse ermittelt und die Potenziale entsprechend reduziert.

\section{Forschungspolitik:}

\section{Kurs Nachbaltigkeit}

ist das Thema

des Informationsdienstes

\section{Ökologisches Wirtschaften 6/04}

Wenn Sie potenzielle Beiträge haben, wenden Sie sich bitte an die Redaktion.
Aus gesetzlich definierten Flächenzielen des Naturschutzes sowie aus naturschutzfachlichen Forderungen wurden dabei von der TU Berlin Flächenansprüche abgeleitet und auf Bundesebene

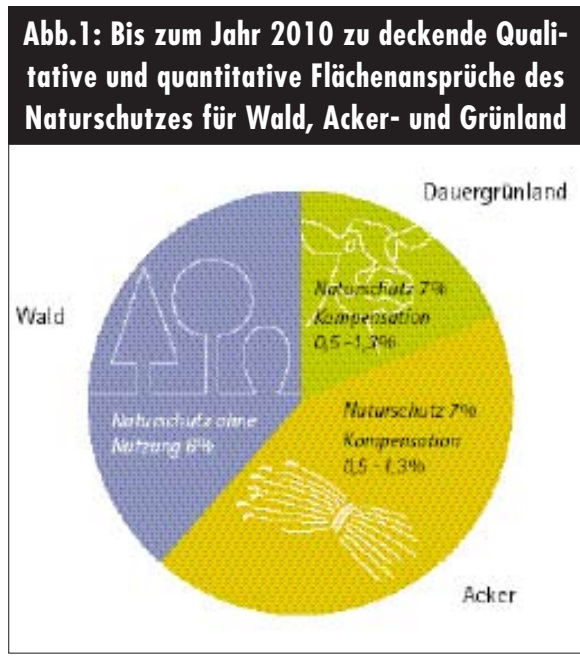

Quelle: siehe Anmerkung 3

abgeschätzt (2). In einer Status-quo-Analyse wurden dann die Flächen ermittelt, die bereits heute vorrangig Naturschutzfunktionen erfüllen und schließlich das zu deckende Defizit gegenüber den gesetzlichen und untergesetzlichen Vorgaben und Zielen des Naturschutzes überschlägig bestimmt (vgl. Abb. 1). Zusätzlich sind Flächen für die naturschutzrechtlich vorgeschriebene Kompensation von Bauvorhaben einzurechnen. Das Ergebnis sind sowohl qualitative als auch quantitative Flächenforderungen des Naturschutzes für Acker- und Grünland, die sukzessive bis zum Jahre 2010 zu erfüllen sind.

Die Studie zeigt, dass die Potenziale der energetischen Biomassenutzung durch die Flächenansprüche des Naturschutzes insgesamt nur geringfügig eingeschränkt werden.

\section{Produktionsbezogene Konfliktpotenziale}

Damit auch die landwirtschaftlich genutzten Flächen Naturschutzfunktionen erfüllen können, sind Anforderungen an die Art und Intensität der Nutzung zu stellen. Biomasse kann durch eine Vielzahl verschiedener Kulturen produziert werden, die ganz unterschiedliche Auswirkungen auf den Naturschutz haben. Einjährige Kulturen, wie Raps oder Mais, fördern beispielsweise wesentlich stärker die Bodenerosion als mehrjährige Energiepflanzen wie Schilf. Die potenziellen Konflikte können durch eine angepasste Auswahl der Anbaukulturen erheblich gemindert werden. Da- 
her muss eine „Gute fachliche Praxis“ speziell für den Anbau von Biomasse weiterentwickelt und an die Ziele des Naturschutzes angepasst werden. Um einen Ausbau der Anbaubiomasse nicht nur unter dem Aspekt , viel Masse = viel Energie = hoher ökonomischer Deckungsbeitrag" zu verstehen, muss der gesamte ökologische Fußabdruck der Biomassebereitstellung in Betracht gezogen werden. Die politische Ableitung daraus muss sein, nur den Biomasseanbau verstärkt zu fördern, der auch einen ökologisch nachhaltigen Ertrag erzielt. Das betrifft auch die Berücksichtigung von Naturschutzzielen durch eine an den Standort und die örtlichen Zielvorgaben angepasste Produktion.

Um entsprechende Anforderungen im Detail formulieren zu können, sind präzise Kenntnisse über die Auswirkungen von Anbaubiomasse auf den Naturhaushalt in all ihren Wechselbeziehungen notwendig. Durch ökologische Wirkungsanalysen können zumindest qualitative Unterschiede erkannt und Umweltkriterien bezüglich der Umweltverträglichkeit benannt werden. Zur naturschutzfachlichen Beurteilung und Einschätzung der dynamischen Entwicklung von Biomasse müssen die mit ihrem Anbau verbundenen ökologischen Wirkfaktoren (Bodenverdichtung, Eintrag von Schadstoffen, Minderung des Erosionswiderstandes, Verschlechterung der Lebensraumfunktion usw.) der verwendeten Kulturen erkannt werden.

Zusätzlich müssen der zeitliche Verlauf der Wirkfaktoren und dadurch entstehende kumulative Wirkungen als Variablen in Betracht gezogen werden. Die meisten einjährigen Kulturen der Anbaubiomasse sind in Fruchtfolgen eingebunden. Um eine umfassendere Wirkungsanalyse zu erstellen, müssen die gesamten Fruchtfolgeketten auf den ökologischen Prüfstand gestellt werden und die komplexen Wechselbeziehungen zwischen den Kulturen berücksichtigt werden. Dies wird zum Beispiel in der Stickstoffbevorratung, der Vorund Nachbehandlung des Standortes durch Pestizide und unterschiedlicher Bodenbearbeitung deutlich. Hier besteht weiterer Forschungsbedarf, um ökologische Bewertungen der gesamten Fruchtfolgekette zu ermöglichen.

\section{- Lösungsstrategien entwickeln}

Zur naturverträglichen Steuerung des verstärkten Anbaus von Biomasse müssen sowohl flächen- als auch produktionsbezogene Lösungsansätze verfolgt werden. Eine optimale Steuerung eines umweltverträglichen Anbaus von Biomasse muss sowohl Standortfaktoren, deren Potenziale und Restriktionen als auch eine ständige Optimierung

\begin{tabular}{|c|c|}
\hline Ausführendes Instrument & Steuerungsmöglichkeit für Anbaubiomasse \\
\hline \multirow[t]{2}{*}{ Cross Compliance Regelung } & Koppelung von höheren ökologischen Standards an Subventionen bei \\
\hline & Bereitstellung von Anbaubiomasse \\
\hline Agrarumweltmaßnahmen & Spezielle Prämien für Anbaubiomasse nach ökologischen Kriterien \\
\hline Flächenstilllegungsrichtlinien & $\begin{array}{l}\text { Differenzierte Flächenstilllegungsprämien (je nach Zielerfüllungsgrad von } \\
\text { naturschutzfachlichen Leitbildern) }\end{array}$ \\
\hline Einspeisevergütungen über Erneuerbare & Besondere Vergütung für Energie aus umweltverträglich produzierter Biomasse \\
\hline Energien Gesetz & \\
\hline
\end{tabular}

der Produktionsbedingungen hinsichtlich naturschutzfachlicher Kriterien berïcksichtigen. Im Konkreten bedeutet dies, dass verschiedene Steuerungsmechanismen auf unterschiedlichen Ebenen die Dynamik des Anbaus beeinflussen müssen. So können einerseits ökonomische Anreize für die gezielte Förderung von gewünschten Kulturen (CO-Kredit, Steuerentlastungen, Prämienzahlungen etc.) sowie regulierende Produktionsauflagen (Gute fachliche Praxis, Cross Compliance, Agrarumweltmaßnahmen etc.) und andererseits die räumliche Steuerung (Landschaftsplanung, Agrarstrukturelle Entwicklungsplanung etc.) zum Einsatz kommen. Es kann eine ortsbezogene vorbereitende planerische Steuerung und eine ortsunabhängige umsetzungsorientierte Steuerung geben, die durch produktionsbezogene Ver- und Gebote eingreift und Produktionsverfahren somit determiniert oder aber durch ökonomische Anreize fördernd wirkt.

Die Steuerungselemente auf verschiedenen Ebenen greifen nur bedingt ineinander. Um gewünschte Effekte zu erzielen, muss sich eine integrale Planung eines Methodenmixes bedienen. Eine Koppelung von wirksamen Instrumenten wird empfohlen. Abbildung 2 zeigt eine Auswahl von potenziellen Steuerungsmöglichkeiten, die zukünftig genutzt werden könnten.

\section{Synergien nutzen}

Zwischen Naturschutzanforderungen und Biomasseanbau können jedoch auch Synergien bestehen. Die Entnahme von Grünschnitt ist teilweise grundlegende Voraussetzung, um Naturschutzziele zu erreichen. So sind beispielsweise viele Hecken und Gebüsche darauf angewiesen, regelmäßig geschnitten zu werden und naturschutzfachlich wertvolles Feuchtgrünland muss gemäht werden. Durch eine energetische Verwertung des Grünschnitts können die Kosten für die Pflegemaßnahmen reduziert werden. Auf bestimmten Standorten kann durch Einsatz von mehrjährigen Energiepflanzen auch die Erosion erheblich gemindert werden.

\section{Fazit}

Eine Integration von Naturschutzzielen in die Biomasseproduktion ist machbar und fördert deren gesellschaftliche Akzeptanz. Die Entwicklung naturund umweltverträglicher Landnutzungssysteme zum Anbau von Biomasse bietet darüber hinaus Möglichkeiten, die Belange des Naturschutzes, des Klimaschutzes und einer nachhaltigen ländlichen Entwicklung zu vereinen. Naturschutz und Bioenergie haben die potenziellen Synergieeffekte bereits in Ansätzen erkannt. Integrierte Systeme bei der Landnutzung und Landschaftspflege berücksichtigen naturschutzfachliche Erfordernisse und bieten einen ökonomischen Ertrag durch energetische Verwertung. Sie sollen Bestandteil von zukünftigen Konzepten sein.

\section{Anmerkungen}

(1) Öko Institut ( $\mathrm{Hg}$.): Stoffstromanalyse zur nachhaltigen energetischen Nutzung von Biomasse - Endbericht. Berlin 2004. Download: www.bmu.de/files/biomasse_vorhaben_endbericht.pdf..

(2) Köppel, J./ Peters, W./ Schultze, C.: Integration naturschutzfachlicher Ziele in Szenarien und Modelle zur energetischen Nutzung von Biomasse. Kurzgutachten zum Umfang der Flächenrestriktionen der energetischen Biomassenutzung durch Naturschutz, Institut für Landschafts- und Umweltplanung TU Berlin, Berlin 2004.

(3) Öko Institut ( $\mathrm{Hg}$.): Stoffstromanalyse zur nachhaltigen energetischen Nutzung von Biomasse. Fact-sheet "Naturschutz, im erscheinen.

\section{Die Autoren}

Dr. Johann Köppel ist Professor, Dr. Wolfgang Peters und Christan Schultze sind wissenschaftliche Mitarbeiter am Institut für Landschafts- und Umweltplanung der TU Berlin.

Kontakt: TU Berlin, Institut für Landschafts- und Umweltplanung, Franklinstr. 28-29, 10587 Berlin. Tel. 030-31473280, E-Mail: koeppel@ile.tu-berlin.de 
(c) 20I0 Authors; licensee IÖW and oekom verlag. This is an article distributed under the terms of the Creative Commons Attribution Non-Commercial No Derivates License (http://creativecommons.org/licenses/by-nc-nd/3.o/), which permits unrestricted use, distribution, and reproduction in any medium, provided the original work is properly cited. 